\title{
Three-Phase Elliptical Inclusions with an Internal Stress Field of Linear Form
}

\author{
Xu Wang ${ }^{1, *}$, Weiqiu Chen ${ }^{2}$ \\ ${ }^{1}$ School of Mechanical and Power Engineering, East China University of Science and Technology, \\ 130 Meilong Road, Shanghai 200237, China \\ ${ }^{2}$ Department of Engineering Mechanics, Zhejiang University, Hangzhou 310027, China \\ * Corresponding author: xuwang@ecust.edu.cn
}

\begin{abstract}
This paper studies the internal stress field of a three-phase elliptical inclusion which is bonded to an infinite matrix through an interphase layer when the matrix is subjected to a linearly distributed in-plane stress field at infinity. Two conditions are found that ensure that the internal non-uniform stress field is simply a linear function of the two coordinates. For given material and geometric parameters of the composite, these conditions can be considered as two restrictions on the applied non-uniform loadings. When these two conditions are met, elementary-form expressions of the stresses in all the three phases are derived. In particular, it is found that the mean stress within the interphase layer is also a linear function of the coordinates. If the interphase layer and the matrix have the same elastic constants, the satisfaction of the two conditions will result in a harmonic inclusion under prescribed non-constant field.
\end{abstract}

Keywords Elliptical inclusion, Interphase layer, Non-uniform loading, Harmonic inclusion, Inverse problem

\section{Introduction}

Rigorous analysis of a composite system consisting of an internal inclusion, an intermediate interphase layer (or coating) and an outer matrix is challenging, especially when the inclusion is non-circular (see for example, [1-5] and the references cited therein). It has been found that the internal stress field within a three-phase confocal elliptical inclusion can be uniform and hydrostatic when the remotely applied uniform in-plane stresses satisfy a condition [2]. How about the stress field within a three-phase elliptical inclusion when the matrix is subjected to non-uniform in-plane stresses at infinity? Is there any elementary solution for the case of non-uniform loading?

In this paper two conditions are found that ensure that the internal stress field within a three-phase confocal elliptical inclusion is a linear function of the coordinates when the matrix is subjected to a linearly distributed (non-uniform) in-plane stress field at infinity. Elementary-form solution is derived when these two conditions are met. Some special examples are presented to demonstrate the solution. In particular, when the interphase layer and the matrix have identical elastic constants, the satisfaction of the two conditions will result in a harmonic elliptical inclusion under non-uniform loadings.

\section{The Internal Stress Field of Linear Form}

We study the stress-field of a three-phase elliptical inclusion with two confocal interfaces when the matrix is subjected to a linearly distributed stress field at infinity. Let $S_{1}, S_{2}$ and $S_{3}$ denote the inclusion, the interphase layer and the matrix, respectively, which are perfectly bonded across two confocal elliptical interfaces $L_{1}$ and $L_{2}$, as shown in Fig. 1 . Throughout the paper, the subscripts 1,2 and 3 are used to identify the respective quantities in $S_{1}, S_{2}$ and $S_{3}$. 


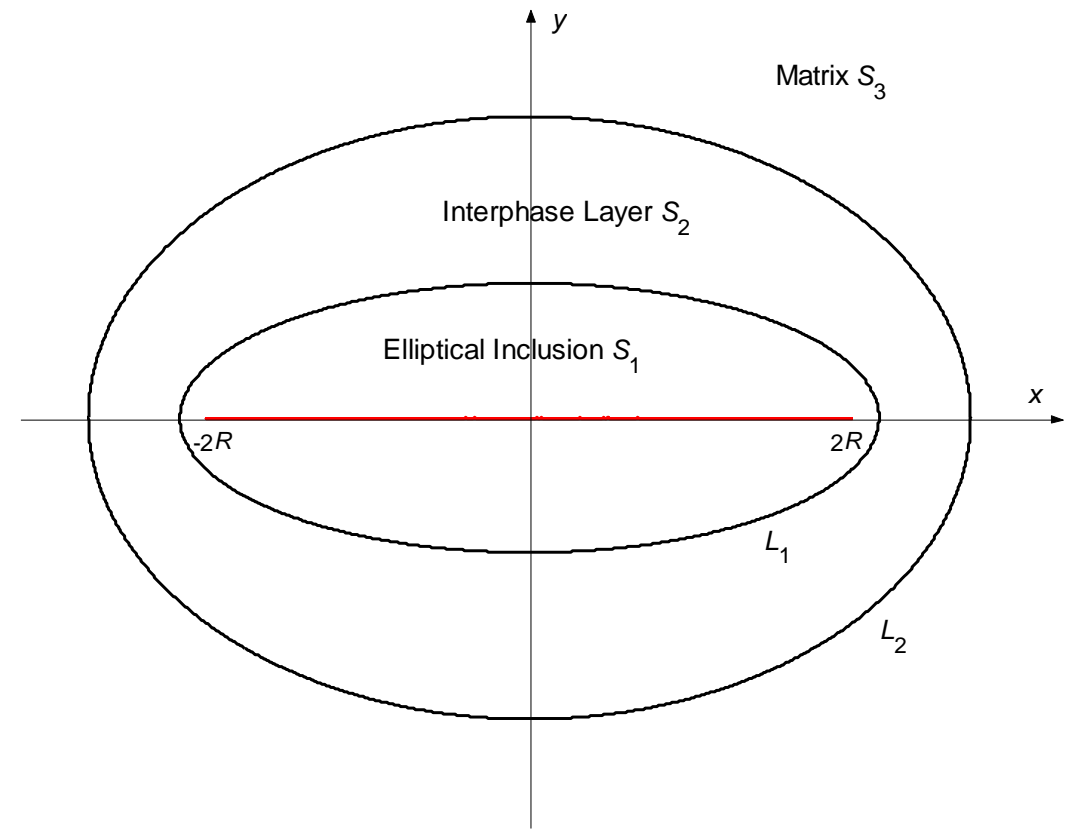

Figure 1 Three-phase elliptical inclusion with an internal stress field of linear form

For plane deformations of an isotropic elastic material, the in-plane displacements $u$ and $v$, the two resultant forces $f_{x}$ and $f_{y}$, and the in-plane stresses $\sigma_{x x}, \sigma_{y y}$ and $\sigma_{x y}$ can be expressed in terms of two analytic functions $\phi(z)$ and $\psi(z)$ of the complex variable $z=x+i y$ as [6]

$$
\begin{aligned}
& 2 \mu(u+\mathrm{i} v)=\kappa \phi(z)-z \overline{\phi^{\prime}(z)}-\overline{\psi(z)}, \\
& f_{x}+\mathrm{i} f_{y}=-\mathrm{i}\left[\phi(z)+z \overline{\phi^{\prime}(z)}+\overline{\psi(z)}\right], \\
& \sigma_{x x}+\sigma_{y y}=2\left[\phi^{\prime}(z)+\overline{\phi^{\prime}(z)}\right], \\
& \sigma_{y y}-\sigma_{x x}+2 \mathrm{i} \sigma_{x y}=2\left[\bar{z} \phi^{\prime \prime}(z)+\psi^{\prime}(z)\right],
\end{aligned}
$$

where $\kappa=3-4 v$ for plane strain, and $\kappa=(3-v) /(1+v)$ for plane stress; $\mu$ and $v$, where $\mu>0$ and $0 \leq v \leq 0.5$, are the shear modulus and Poisson's ratio, respectively.

In the physical z-plane, the boundary value problem for the three-phase elliptical inclusion takes the form:

$$
\begin{aligned}
& \phi_{2}(z)+z \overline{\phi_{2}^{\prime}(z)}+\overline{\psi_{2}(z)}=\phi_{1}(z)+z \overline{\phi_{1}^{\prime}(z)}+\overline{\psi_{1}(z)} \\
& \frac{1}{2 \mu_{2}}\left[\kappa_{2} \phi_{2}(z)-z \overline{\phi_{2}^{\prime}(z)}-\overline{\psi_{2}(z)}\right]=\frac{1}{2 \mu_{1}}\left[\kappa_{1} \phi_{1}(z)-z \overline{\phi_{1}^{\prime}(z)}-\overline{\psi_{1}(z)}\right], \quad z \in L_{1} ; \\
& \phi_{2}(z)+z \overline{\phi_{2}^{\prime}(z)}+\overline{\psi_{2}(z)}=\phi_{3}(z)+z \overline{\phi_{3}^{\prime}(z)}+\overline{\psi_{3}(z)}, \\
& \frac{1}{2 \mu_{2}}\left[\kappa_{2} \phi_{2}(z)-z \overline{\phi_{2}^{\prime}(z)}-\overline{\psi_{2}(z)}\right]=\frac{1}{2 \mu_{3}}\left[\kappa_{3} \phi_{3}(z)-z \overline{\phi_{3}^{\prime}(z)}-\overline{\psi_{3}(z)}\right] \quad z \in L_{2} ; \\
& \phi_{3}(z) \cong\left(A_{1}+\mathrm{i} A_{2}\right) z^{2}+O(1), \quad \psi_{3}(z) \cong\left(B_{1}+i B_{2}\right) z^{2}+O(1), \quad \text { as } \quad|z| \rightarrow \infty
\end{aligned}
$$

where $A_{1}, A_{2}, B_{1}$ and $B_{2}$ are real numbers related to the applied linearly distributed stress field at infinity. 
Consider the following conformal mapping $[2,6]$

$$
z=\omega(\xi)=R\left(\xi+\frac{1}{\xi}\right), \quad \xi(z)=\frac{z}{2 R}\left[1+\sqrt{1-\frac{4 R^{2}}{z^{2}}}\right], \quad(|\xi| \geq 1)
$$

which maps the segment $[-2 R 2 R]$ onto the unit circle in the $\xi$-plane, and the two interfaces $L_{1}$ and $L_{2}$ are mapped onto two coaxial circles with radii $R_{1}$ and $R_{2}$, respectively. Thus the three regions $S_{1}$, $S_{2}$ and $S_{3}$ are mapped onto the annuli $1<|\xi|<R_{1}, \quad R_{1}<|\xi|<R_{2}$ and $|\xi|>R_{2}$, respectively, as shown in Fig. 2. For convenience we will write $\phi_{i}(\xi)=\phi_{i}(\omega(\xi)), \psi_{i}(\xi)=\psi_{i}(\omega(\xi)), i=1,2,3$.

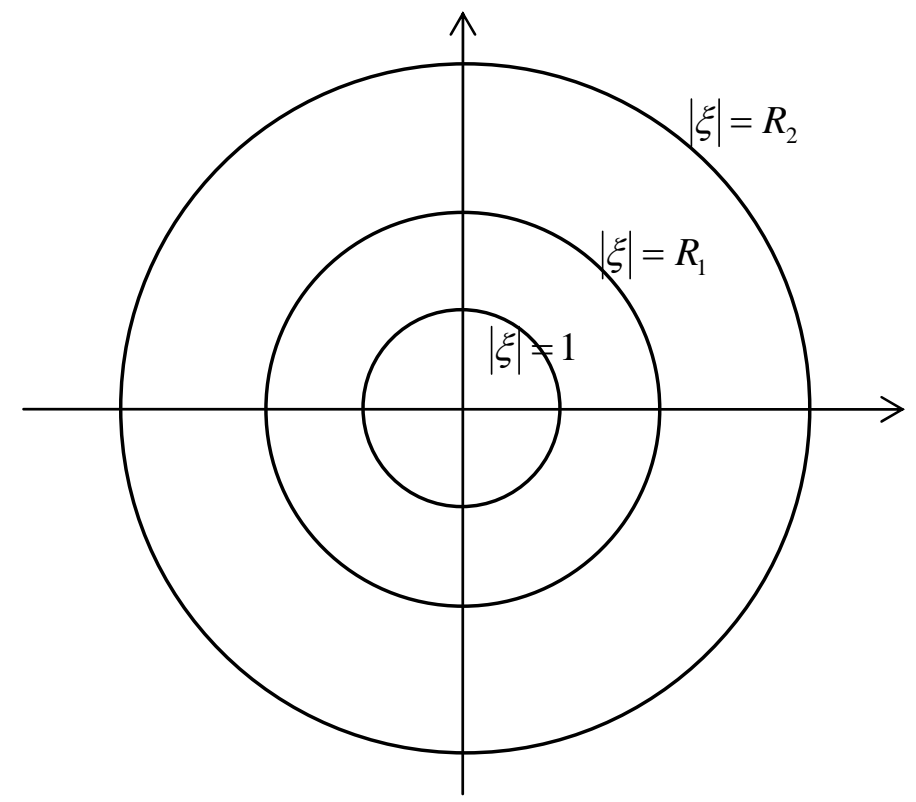

Figure 2 The mapped $\xi$-plane

In the mapped $\xi$-plane, the boundary value problem takes the form

$$
\begin{aligned}
& \phi_{2}(\xi)+\omega(\xi) \frac{\overline{\phi_{2}^{\prime}(\xi)}}{\overline{\omega^{\prime}(\xi)}}+\overline{\psi_{2}(\xi)}=\phi_{1}(\xi)+\omega(\xi) \frac{\overline{\phi_{1}^{\prime}(\xi)}}{\overline{\omega^{\prime}(\xi)}}+\overline{\psi_{1}(\xi),} \\
& \kappa_{2} \phi_{2}(\xi)-\omega(\xi) \frac{\overline{\phi_{2}^{\prime}(\xi)}}{\overline{\omega^{\prime}(\xi)}}-\overline{\psi_{2}(\xi)}=\frac{\kappa_{1}}{\Gamma_{1}} \phi_{1}(\xi)-\frac{1}{\Gamma_{1}} \omega(\xi) \frac{\overline{\phi_{1}^{\prime}(\xi)}}{\overline{\omega^{\prime}(\xi)}}-\frac{1}{\Gamma_{1}} \overline{\psi_{1}(\xi)}, \\
& \phi_{3}(\xi)+\omega(\xi) \frac{\overline{\phi_{3}^{\prime}(\xi)}}{\overline{\omega^{\prime}(\xi)}}+\overline{\psi_{3}(\xi)}=\phi_{2}(\xi)+\omega(\xi) \frac{\overline{\phi_{2}^{\prime}(\xi)}}{\overline{\omega^{\prime}(\xi)}}+\overline{\psi_{2}(\xi)}, \quad \text { on }|\xi|=R_{1} \\
& \kappa_{3} \phi_{3}(\xi)-\omega(\xi) \frac{\overline{\phi_{3}^{\prime}(\xi)}}{\overline{\omega^{\prime}(\xi)}}-\overline{\psi_{3}(\xi)}=\Gamma_{3} \kappa_{2} \phi_{2}(\xi)-\Gamma_{3} \omega(\xi) \frac{\overline{\phi_{2}^{\prime}(\xi)}}{\frac{\omega^{\prime}(\xi)}{\Gamma_{3}}} \overline{\psi_{2}(\xi),} \\
& \phi_{3}(\xi) \cong R^{2}\left(A_{1}+\mathrm{i} A_{2}\right) \xi^{2}+O(1), \quad \psi_{3}(\xi) \cong R^{2}\left(B_{1}+\mathrm{i} B_{2}\right) \xi^{2}+O(1), \quad \text { as } \quad|\xi|=R_{2}
\end{aligned}
$$

where $\Gamma_{1}=\mu_{1} / \mu_{2}$ and $\Gamma_{3}=\mu_{3} / \mu_{2}$ are two stiffness ratios.

In order to ensure that the internal stress field is a linear function of the coordinates $x$ and $y, \phi_{1}(\xi)$ and $\psi_{1}(\xi)$ must take the following forms 


$$
\phi_{1}(\xi)=X\left(\xi^{2}+\frac{1}{\xi^{2}}\right), \quad \psi_{1}(\xi)=Y\left(\xi^{2}+\frac{1}{\xi^{2}}\right), \quad\left(1 \leq|\xi| \leq R_{1}\right)
$$

where $X$ and $Y$ are complex constants to be determined.

Consequently it follows from Eqs. (7) and (10) that

$$
\begin{array}{r}
\left(\kappa_{2}+1\right) \phi_{2}(\xi)=\frac{X\left(\Gamma_{1}+\kappa_{1}\right)}{\Gamma_{1}}\left(\xi^{2}+\frac{1}{\xi^{2}}\right)+\frac{2 \bar{X}\left(\Gamma_{1}-1\right)}{\Gamma_{1}}\left(\frac{\xi^{2}}{R_{1}^{2}}+\frac{R_{1}^{2}}{\xi^{2}}\right)+\frac{\bar{Y}\left(\Gamma_{1}-1\right)}{\Gamma_{1}}\left(\frac{\xi^{2}}{R_{1}^{4}}+\frac{R_{1}^{4}}{\xi^{2}}\right), \\
\left(R_{1} \leq|\xi| \leq R_{2}\right)
\end{array}
$$

It is found that the necessary and sufficient condition for the validity of Eq. (10) for the internal stress field is that $\phi_{2}(\xi)$ should take the form of $\phi_{2}(\xi)=\lambda\left(\xi^{2}+1 / \xi^{2}\right)$ with $\lambda$ being a complex constant. This condition can be easily arrived at from Eq. (2.14) in [2]. The necessity is true only when $\rho=R_{1}^{2} / R_{2}^{2} \neq 1$ and $\Gamma_{3} \neq 1$. Consequently the following relationship between $X$ and $Y$ establishes

$$
Y=-\frac{2 X}{R_{1}^{2}+R_{1}^{-2}}, \quad\left(\rho \neq 1, \Gamma_{1} \neq 1, \Gamma_{3} \neq 1\right)
$$

Once the above relationship is satisfied, and all the interface conditions in Eqs. (7) are enforced, $\phi_{2}(\xi)$ and $\psi_{2}(\xi)$ are found to have the following expressions

$$
\begin{aligned}
\phi_{2}(\xi)= & \frac{X\left(\Gamma_{1}+\kappa_{1}\right)\left(R_{1}^{2}+R_{1}^{-2}\right)+2 \bar{X}\left(\Gamma_{1}-1\right)}{\Gamma_{1}\left(\kappa_{2}+1\right)\left(R_{1}^{2}+R_{1}^{-2}\right)}\left(\xi^{2}+\frac{1}{\xi^{2}}\right) \\
\psi_{2}(\xi)= & \frac{2 X\left[R_{1}^{-4}\left[\Gamma_{1}\left(\kappa_{2}-1\right)-\left(\kappa_{1}-1\right)\right]-\Gamma_{1}-\kappa_{1}\right]+\bar{X}\left[R_{1}^{-2}\left[4-\kappa_{1}-\Gamma_{1}\left(4-\kappa_{2}\right)\right]+R_{1}^{-6}\left(\Gamma_{1} \kappa_{2}-\kappa_{1}\right)\right]}{\Gamma_{1}\left(\kappa_{2}+1\right)\left(R_{1}^{2}+R_{1}^{-2}\right)} \xi^{2} \\
& +\frac{2 X\left[R_{1}^{4}\left[\Gamma_{1}\left(\kappa_{2}-1\right)-\left(\kappa_{1}-1\right)\right]-\Gamma_{1}-\kappa_{1}\right]+\bar{X}\left[R_{1}^{2}\left[4-\kappa_{1}-\Gamma_{1}\left(4-\kappa_{2}\right)\right]+R_{1}^{6}\left(\Gamma_{1} \kappa_{2}-\kappa_{1}\right)\right]}{\Gamma_{1}\left(\kappa_{2}+1\right)\left(R_{1}^{2}+R_{1}^{-2}\right)} \frac{1}{\xi^{2}}
\end{aligned}
$$

Similarly by enforcing the interface conditions in Eq. (8), we arrive at the following expression of $\phi_{3}(\xi)$ and $\psi_{3}(\xi)$

$$
\begin{aligned}
& \phi_{3}(\xi) \\
& =\frac{\left(\begin{array}{l}
\left(\Gamma_{3} \kappa_{2}+1\right)\left[X\left(\Gamma_{1}+\kappa_{1}\right)\left(R_{1}^{2}+R_{1}^{-2}\right)+2 \bar{X}\left(\Gamma_{1}-1\right)\right]\left(\xi^{2}+\frac{1}{\xi^{2}}\right) \\
+2\left(1-\Gamma_{3}\right)\left[\bar{X}\left(\Gamma_{1}+\kappa_{1}\right)\left(R_{1}^{2}+R_{1}^{-2}\right)+2 X\left(\Gamma_{1}-1\right)\left(\frac{\xi^{2}}{R_{2}^{2}}+\frac{R_{2}^{2}}{\xi^{2}}\right)\right. \\
+R_{2}^{-4}\left(1-\Gamma_{3}\right)\left\{2 \bar{X}\left[R_{1}^{4}\left[\Gamma_{1}\left(\kappa_{2}-1\right)-\left(\kappa_{1}-1\right)\right]-\Gamma_{1}-\kappa_{1}\right]+X\left[R_{1}^{2}\left[4-\kappa_{1}-\Gamma_{1}\left(4-\kappa_{2}\right)\right]+R_{1}^{6}\left(\Gamma_{1} \kappa_{2}-\kappa_{1}\right)\right]\right\} \xi^{2} \\
+R_{2}^{4}\left(1-\Gamma_{3}\right)\left\{2 \bar{X}\left[R_{1}^{-4}\left[\Gamma_{1}\left(\kappa_{2}-1\right)-\left(\kappa_{1}-1\right)\right]-\Gamma_{1}-\kappa_{1}\right]+X\left[R_{1}^{-2}\left[4-\kappa_{1}-\Gamma_{1}\left(4-\kappa_{2}\right)\right]+R_{1}^{-6}\left(\Gamma_{1} \kappa_{2}-\kappa_{1}\right)\right]\right\} \frac{1}{\xi^{2}}
\end{array}\right.}{\Gamma_{1}\left(\kappa_{2}+1\right)\left(\kappa_{3}+1\right)\left(R_{1}^{2}+R_{1}^{-2}\right)},
\end{aligned}
$$$$
\left(|\xi| \geq R_{2}\right)
$$ 
$\psi_{3}(\xi)$

$$
\begin{aligned}
& \left(\begin{array}{l}
\left(\kappa_{3}-\Gamma_{3} \kappa_{2}\right)\left[\bar{X}\left(\Gamma_{1}+\kappa_{1}\right)\left(R_{1}^{2}+R_{1}^{-2}\right)+2 X\left(\Gamma_{1}-1\right)\left(\frac{\xi^{2}}{R_{2}^{4}}+\frac{R_{2}^{4}}{\xi^{2}}\right)\right. \\
\left.+2\left(\kappa_{3}+\Gamma_{3}\right)\left[X\left(\Gamma_{1}+\kappa_{1}\right)\left(R_{1}^{2}+R_{1}^{-2}\right)+2 \bar{X}\left(\Gamma_{1}-1\right)\right] \frac{\xi^{2}}{R_{2}^{2}}+\frac{R_{2}^{2}}{\xi^{2}}\right) \\
+\left(\Gamma_{3}+\kappa_{3}\right)\left\{2 X\left[R_{1}^{-4}\left[\Gamma_{1}\left(\kappa_{2}-1\right)-\left(\kappa_{1}-1\right)\right]-\Gamma_{1}-\kappa_{1}\right]+\bar{X}\left[R_{1}^{-2}\left[4-\kappa_{1}-\Gamma_{1}\left(4-\kappa_{2}\right)\right]+R_{1}^{-6}\left(\Gamma_{1} \kappa_{2}-\kappa_{1}\right)\right]\right\} \xi^{2} \\
+\left(\Gamma_{3}+\kappa_{3}\right)\left\{2 X\left[R_{1}^{4}\left[\Gamma_{1}\left(\kappa_{2}-1\right)-\left(\kappa_{1}-1\right)\right]-\Gamma_{1}-\kappa_{1}\right]+\bar{X}\left[R_{1}^{2}\left[4-\kappa_{1}-\Gamma_{1}\left(4-\kappa_{2}\right)\right]+R_{1}^{6}\left(\Gamma_{1} \kappa_{2}-\kappa_{1}\right)\right] \frac{1}{\xi^{2}}\right.
\end{array}\right) \\
& =\frac{2\left(\frac{\xi}{R_{2}^{2}}+\frac{R_{2}^{2}}{\xi}\right)}{\xi-\frac{1}{\xi}}
\end{aligned}
$$

$$
\left(\begin{array}{l}
\left(\Gamma_{3} \kappa_{2}+1\right)\left[X\left(\Gamma_{1}+\kappa_{1}\right)\left(R_{1}^{2}+R_{1}^{-2}\right)+2 \bar{X}\left(\Gamma_{1}-1\right)\right]\left(\xi^{2}-\frac{1}{\xi^{2}}\right) \\
+2\left(1-\Gamma_{3}\right)\left[\bar{X}\left(\Gamma_{1}+\kappa_{1}\right)\left(R_{1}^{2}+R_{1}^{-2}\right)+2 X\left(\Gamma_{1}-1\right)\left(\frac{\xi^{2}}{R_{2}^{2}}-\frac{R_{2}^{2}}{\xi^{2}}\right)\right. \\
+R_{2}^{-4}\left(1-\Gamma_{3}\right)\left\{2 \bar{X}\left[R_{1}^{4}\left[\Gamma_{1}\left(\kappa_{2}-1\right)-\left(\kappa_{1}-1\right)\right]-\Gamma_{1}-\kappa_{1}\right]+X\left[R_{1}^{2}\left[4-\kappa_{1}-\Gamma_{1}\left(4-\kappa_{2}\right)\right]+R_{1}^{6}\left(\Gamma_{1} \kappa_{2}-\kappa_{1}\right)\right]\right\} \xi^{2} \\
+R_{2}^{4}\left(\Gamma_{3}-1\right)\left\{2 \bar{X}\left[R_{1}^{-4}\left[\Gamma_{1}\left(\kappa_{2}-1\right)-\left(\kappa_{1}-1\right)\right]-\Gamma_{1}-\kappa_{1}\right]+X\left[R_{1}^{-2}\left[4-\kappa_{1}-\Gamma_{1}\left(4-\kappa_{2}\right)\right]+R_{1}^{-6}\left(\Gamma_{1} \kappa_{2}-\kappa_{1}\right)\right]\right\} \frac{1}{\xi^{2}}
\end{array}\right),
$$

$\left(|\xi| \geq R_{2}\right)$

In addition the satisfaction of the remote boundary condition in Eq. (9) will yield $R^{2}\left(A_{1}+\mathrm{i} A_{2}\right)$

$$
=\frac{\left(\begin{array}{l}
\left(\Gamma_{3} \kappa_{2}+1\right)\left[X\left(\Gamma_{1}+\kappa_{1}\right)\left(R_{1}^{2}+R_{1}^{-2}\right)+2 \bar{X}\left(\Gamma_{1}-1\right)\right]+2 R_{2}^{-2}\left(1-\Gamma_{3}\right)\left[\bar{X}\left(\Gamma_{1}+\kappa_{1}\right)\left(R_{1}^{2}+R_{1}^{-2}\right)+2 X\left(\Gamma_{1}-1\right)\right] \\
+R_{2}^{-4}\left(1-\Gamma_{3}\right)\left\{2 \bar{X}\left[R_{1}^{4}\left[\Gamma_{1}\left(\kappa_{2}-1\right)-\left(\kappa_{1}-1\right)\right]-\Gamma_{1}-\kappa_{1}\right]+X\left[R_{1}^{2}\left[4-\kappa_{1}-\Gamma_{1}\left(4-\kappa_{2}\right)\right]+R_{1}^{6}\left(\Gamma_{1} \kappa_{2}-\kappa_{1}\right)\right]\right\}
\end{array}\right)}{\Gamma_{1}\left(\kappa_{2}+1\right)\left(\kappa_{3}+1\right)\left(R_{1}^{2}+R_{1}^{-2}\right)},
$$

$$
\begin{aligned}
& R^{2}\left(B_{1}+\mathrm{i} B_{2}\right) \\
& =\left(\begin{array}{l}
R_{2}^{-4}\left[\kappa_{3}-4+\Gamma_{3}\left(4-\kappa_{2}\right)\right]\left[\bar{X}\left(\Gamma_{1}+\kappa_{1}\right)\left(R_{1}^{2}+R_{1}^{-2}\right)+2 X\left(\Gamma_{1}-1\right)\right] \\
+2 R_{2}^{-2}\left[\kappa_{3}-1+\Gamma_{3}\left(1-\kappa_{2}\right)\right]\left[X\left(\Gamma_{1}+\kappa_{1}\right)\left(R_{1}^{2}+R_{1}^{-2}\right)+2 \bar{X}\left(\Gamma_{1}-1\right)\right] \\
+\left(\Gamma_{3}+\kappa_{3}\right)\left\{2 X\left[R_{1}^{-4}\left[\Gamma_{1}\left(\kappa_{2}-1\right)-\left(\kappa_{1}-1\right)\right]-\Gamma_{1}-\kappa_{1}\right]+\bar{X}\left[R_{1}^{-2}\left[4-\kappa_{1}-\Gamma_{1}\left(4-\kappa_{2}\right)\right]+R_{1}^{-6}\left(\Gamma_{1} \kappa_{2}-\kappa_{1}\right)\right]\right\} \\
+2 R_{2}^{-6}\left(\Gamma_{3}-1\right)\left\{2 \bar{X}\left[R_{1}^{4}\left[\Gamma_{1}\left(\kappa_{2}-1\right)-\left(\kappa_{1}-1\right)\right]-\Gamma_{1}-\kappa_{1}\right]+X\left[R_{1}^{2}\left[4-\kappa_{1}-\Gamma_{1}\left(4-\kappa_{2}\right)\right]+R_{1}^{6}\left(\Gamma_{1} \kappa_{2}-\kappa_{1}\right)\right]\right\}
\end{array}\right) . \\
& \Gamma_{1}\left(\kappa_{2}+1\right)\left(\kappa_{3}+1\right)\left(R_{1}^{2}+R_{1}^{-2}\right)
\end{aligned}
$$


It turns out that the necessary and sufficient condition for the existence of the complex coefficient $X$ simultaneously satisfying Eqs. (16) and (17) is:

$$
\frac{B_{1}}{A_{1}}=f\left(R_{1}, \rho\right), \quad \frac{B_{2}}{A_{2}}=g\left(R_{1}, \rho\right)
$$

where the two functions $f\left(R_{1}, \rho\right)$ and $g\left(R_{1}, \rho\right)$ are defined by

$$
\begin{aligned}
& f\left(R_{1}, \rho\right) \\
& \left(\begin{array}{l}
\left\{\rho^{2} R_{1}^{-4}\left[\kappa_{3}-4+\Gamma_{3}\left(4-\kappa_{2}\right)\right]+2 \rho R_{1}^{-2}\left[\kappa_{3}-1+\Gamma_{3}\left(1-\kappa_{2}\right)\right]\right\}\left[\left(\Gamma_{1}+\kappa_{1}\right)\left(R_{1}^{2}+R_{1}^{-2}\right)+2\left(\Gamma_{1}-1\right)\right] \\
+\left(\Gamma_{3}+\kappa_{3}\right)\left\{R_{1}^{-6}\left(\Gamma_{1} \kappa_{2}-\kappa_{1}\right)+2 R_{1}^{-4}\left[\Gamma_{1}\left(\kappa_{2}-1\right)-\left(\kappa_{1}-1\right)\right]+R_{1}^{-2}\left[4-\kappa_{1}-\Gamma_{1}\left(4-\kappa_{2}\right)\right]-2\left(\Gamma_{1}+\kappa_{1}\right)\right\} \\
+2 \rho^{3}\left(\Gamma_{3}-1\right)\left\{\left(\Gamma_{1} \kappa_{2}-\kappa_{1}\right)+2 R_{1}^{-2}\left[\Gamma_{1}\left(\kappa_{2}-1\right)-\left(\kappa_{1}-1\right)\right]+R_{1}^{-4}\left[4-\kappa_{1}-\Gamma_{1}\left(4-\kappa_{2}\right)\right]-2 R_{1}^{-6}\left(\Gamma_{1}+\kappa_{1}\right)\right\}
\end{array}\right), \\
& =\frac{\left(\begin{array}{l}
\left.\left[\Gamma_{3} \kappa_{2}+1\right)+2 \rho R_{1}^{-2}\left(1-\Gamma_{3}\right)\right]\left[\left(\Gamma_{1}+\kappa_{1}\right)\left(R_{1}^{2}+R_{1}^{-2}\right)+2\left(\Gamma_{1}-1\right)\right] \\
+\rho^{2}\left(1-\Gamma_{3}\right)\left\{R_{1}^{2}\left(\Gamma_{1} \kappa_{2}-\kappa_{1}\right)+2\left[\Gamma_{1}\left(\kappa_{2}-1\right)-\left(\kappa_{1}-1\right)\right]+R_{1}^{-2}\left[4-\kappa_{1}-\Gamma_{1}\left(4-\kappa_{2}\right)\right]-2 R_{1}^{-4}\left(\Gamma_{1}+\kappa_{1}\right)\right\}
\end{array}\right)}{g\left(R_{1}, \rho\right)} \\
& \left.\qquad \begin{array}{l}
+\left\{2 \rho R_{1}^{-2}\left[\kappa_{3}-1+\Gamma_{3}\left(1-\kappa_{2}\right)\right]-\rho^{2} R_{1}^{-4}\left[\kappa_{3}-4+\Gamma_{3}\left(4-\kappa_{2}\right)\right]\right\}\left[\left(\Gamma_{1}+\kappa_{1}\right)\left(R_{1}^{2}+R_{1}^{-2}\right)-2\left(\Gamma_{1}-1\right)\right] \\
+\left(\Gamma_{3}+\kappa_{3}\right)\left\{R_{1}^{-6}\left(\kappa_{1}-\Gamma_{1} \kappa_{2}\right)+2 R_{1}^{-4}\left[\Gamma_{1}\left(\kappa_{2}-1\right)-\left(\kappa_{1}-1\right)\right]+R_{1}^{-2}\left[\Gamma_{1}\left(4-\kappa_{2}\right)-\left(4-\kappa_{1}\right)\right]-2\left(\Gamma_{1}+\kappa_{1}\right)\right\} \\
+2 \rho^{3}\left(\Gamma_{3}-1\right)\left\{\left(\Gamma_{1} \kappa_{2}-\kappa_{1}\right)+2 R_{1}^{-2}\left[\kappa_{1}-1-\Gamma_{1}\left(\kappa_{2}-1\right)\right]+R_{1}^{-4}\left[4-\kappa_{1}-\Gamma_{1}\left(4-\kappa_{2}\right)\right]+2 R_{1}^{-6}\left(\Gamma_{1}+\kappa_{1}\right)\right\}
\end{array}\right) \\
& \left(\begin{array}{l}
\left.\left[\Gamma_{3} \kappa_{2}+1+2 \rho R_{1}^{-2}\left(\Gamma_{3}-1\right)\right]\left(\Gamma_{1}+\kappa_{1}\right)\left(R_{1}^{2}+R_{1}^{-2}\right)-2\left(\Gamma_{1}-1\right)\right] \\
+\rho^{2}\left(1-\Gamma_{3}\right)\left\{R_{1}^{2}\left(\Gamma_{1} \kappa_{2}-\kappa_{1}\right)+2\left[\kappa_{1}-1-\Gamma_{1}\left(\kappa_{2}-1\right)\right]+R_{1}^{-2}\left[4-\kappa_{1}-\Gamma_{1}\left(4-\kappa_{2}\right)\right]+2 R_{1}^{-4}\left(\Gamma_{1}+\kappa_{1}\right)\right\}
\end{array}\right)
\end{aligned}
$$

If the two conditions in Eq. (18) are met, the internal stress field of linear form within the elliptical inclusion is given by

$$
\begin{aligned}
& \phi_{1}(z)=\frac{X}{R^{2}} z^{2}, \quad \psi_{1}(z)=-\frac{2 X}{R^{2}\left(R_{1}^{2}+R_{1}^{-2}\right)} z^{2}, \\
& \sigma_{x x}=\frac{2\left(R_{1}+R_{1}^{-1}\right)^{2} X_{1} x-2\left(3 R_{1}^{2}+3 R_{1}^{-2}+2\right) X_{2} y}{R^{2}\left(R_{1}^{2}+R_{1}^{-2}\right)}, \\
& \sigma_{y y}=\frac{2\left(3 R_{1}^{2}+3 R_{1}^{-2}-2\right) X_{1} x-2\left(R_{1}-R_{1}^{-1}\right)^{2} X_{2} y}{R^{2}\left(R_{1}^{2}+R_{1}^{-2}\right)}, \quad z \in S_{1} \\
& \sigma_{x y}=\frac{2\left(R_{1}-R_{1}^{-1}\right)^{2} X_{2} X-2\left(R_{1}+R_{1}^{-1}\right)^{2} X_{1} y}{R^{2}\left(R_{1}^{2}+R_{1}^{-2}\right)},
\end{aligned}
$$

where $X_{1}$ and $X_{2}$ are the real and imaginary parts of the complex number $X$, which is determined by either Eq. (16) or Eq. (17). For example, it follows from Eq. (16) that 


$$
\begin{aligned}
& X_{1}=\frac{R^{2} A_{1} \Gamma_{1}\left(\kappa_{2}+1\right)\left(\kappa_{3}+1\right)\left(R_{1}^{2}+R_{1}^{-2}\right)}{\left(\begin{array}{l}
\left(\Gamma_{3} \kappa_{2}+1\right)\left[\left(\Gamma_{1}+\kappa_{1}\right)\left(R_{1}^{2}+R_{1}^{-2}\right)+2\left(\Gamma_{1}-1\right)\right]+2 R_{2}^{-2}\left(1-\Gamma_{3}\right)\left[\left(\Gamma_{1}+\kappa_{1}\right)\left(R_{1}^{2}+R_{1}^{-2}\right)+2\left(\Gamma_{1}-1\right)\right] \\
+R_{2}^{-4}\left(1-\Gamma_{3}\right)\left\{R_{1}^{6}\left(\Gamma_{1} \kappa_{2}-\kappa_{1}\right)+2 R_{1}^{4}\left[\Gamma_{1}\left(\kappa_{2}-1\right)-\left(\kappa_{1}-1\right)\right]+R_{1}^{2}\left[4-\kappa_{1}-\Gamma_{1}\left(4-\kappa_{2}\right)\right]-2\left(\Gamma_{1}+\kappa_{1}\right)\right\}
\end{array}\right)}, \\
& X_{2}=\frac{R^{2} A_{2} \Gamma_{1}\left(\kappa_{2}+1\right)\left(\kappa_{3}+1\right)\left(R_{1}^{2}+R_{1}^{-2}\right)}{\left(\begin{array}{l}
\left(\Gamma_{3} \kappa_{2}+1\right)\left[\left(\Gamma_{1}+\kappa_{1}\right)\left(R_{1}^{2}+R_{1}^{-2}\right)-2\left(\Gamma_{1}-1\right)\right]+2 R_{2}^{-2}\left(\Gamma_{3}-1\right)\left[\left(\Gamma_{1}+\kappa_{1}\right)\left(R_{1}^{2}+R_{1}^{-2}\right)+2\left(1-\Gamma_{1}\right)\right] \\
+R_{2}^{-4}\left(1-\Gamma_{3}\right)\left\{R_{1}^{6}\left(\Gamma_{1} \kappa_{2}-\kappa_{1}\right)-2 R_{1}^{4}\left[\Gamma_{1}\left(\kappa_{2}-1\right)-\left(\kappa_{1}-1\right)\right]+R_{1}^{2}\left[4-\kappa_{1}-\Gamma_{1}\left(4-\kappa_{2}\right)\right]+2\left(\Gamma_{1}+\kappa_{1}\right)\right\}
\end{array}\right)} .
\end{aligned}
$$

Furthermore it is deduced from Eqs. (2) and (13) 1 that the mean stress is linearly distributed within the interphase layer as

$$
\begin{aligned}
& \phi_{2}(z)=\frac{X\left(\Gamma_{1}+\kappa_{1}\right)\left(R_{1}^{2}+R_{1}^{-2}\right)+2 \bar{X}\left(\Gamma_{1}-1\right)}{R^{2} \Gamma_{1}\left(\kappa_{2}+1\right)\left(R_{1}^{2}+R_{1}^{-2}\right)} z^{2}, \\
& \sigma_{x x}+\sigma_{y y}=\frac{8\left[\left(\Gamma_{1}+\kappa_{1}\right)\left(R_{1}^{2}+R_{1}^{-2}\right)+2\left(\Gamma_{1}-1\right)\right] X_{1} x-8\left[\left(\Gamma_{1}+\kappa_{1}\right)\left(R_{1}^{2}+R_{1}^{-2}\right)-2\left(\Gamma_{1}-1\right)\right] X_{2} y}{R^{2} \Gamma_{1}\left(\kappa_{2}+1\right)\left(R_{1}^{2}+R_{1}^{-2}\right)},
\end{aligned}
$$

By making use of Eqs. (16) and (17), $\phi_{3}(\xi)$ and $\psi_{3}(\xi)$ in Eqs. (14) and (15) can be re-expressed into

$$
\begin{aligned}
& \phi_{3}(\xi)=R^{2}\left(A_{1}+\mathrm{i} A_{2}\right) \xi^{2} \\
& +\frac{\left(\begin{array}{l}
\left(\Gamma_{3} \kappa_{2}+1\right)\left[X\left(\Gamma_{1}+\kappa_{1}\right)\left(R_{1}^{2}+R_{1}^{-2}\right)+2 \bar{X}\left(\Gamma_{1}-1\right)\right]+2 R_{2}^{2}\left(1-\Gamma_{3}\right)\left[\bar{X}\left(\Gamma_{1}+\kappa_{1}\right)\left(R_{1}^{2}+R_{1}^{-2}\right)+2 X\left(\Gamma_{1}-1\right)\right] \\
+R_{2}^{4}\left(1-\Gamma_{3}\right)\left\{2 \bar{X}\left[R_{1}^{-4}\left[\Gamma_{1}\left(\kappa_{2}-1\right)-\left(\kappa_{1}-1\right)\right]-\Gamma_{1}-\kappa_{1}\right]+X\left[R_{1}^{-2}\left[4-\kappa_{1}-\Gamma_{1}\left(4-\kappa_{2}\right)\right]+R_{1}^{-6}\left(\Gamma_{1} \kappa_{2}-\kappa_{1}\right)\right]\right\}
\end{array}\right)}{\Gamma_{1}\left(\kappa_{2}+1\right)\left(\kappa_{3}+1\right)\left(R_{1}^{2}+R_{1}^{-2}\right) \xi^{2}},
\end{aligned}
$$$$
\left(|\xi| \geq R_{2}\right)
$$

$$
\begin{aligned}
& \psi_{3}(\xi)=R^{2}\left(B_{1}+\mathrm{i} B_{2}\right) \xi^{2}-\frac{2\left[\alpha\left(R_{2}^{2}+R_{2}^{-2}\right)+\beta R_{2}^{-2}\right] \xi^{2}+2 \beta R_{2}^{2}}{\Gamma_{1}\left(\kappa_{2}+1\right)\left(\kappa_{3}+1\right)\left(R_{1}^{2}+R_{1}^{-2}\right) \xi^{2}\left(\xi^{2}-1\right)} \\
& +\frac{\left.\begin{array}{l}
R_{2}^{4}\left(\kappa_{3}-\Gamma_{3} \kappa_{2}\right)\left[\bar{X}\left(\Gamma_{1}+\kappa_{1}\right)\left(R_{1}^{2}+R_{1}^{-2}\right)+2 X\left(\Gamma_{1}-1\right)\right] \\
+2 R_{2}^{2}\left(\kappa_{3}+\Gamma_{3}\right)\left[X\left(\Gamma_{1}+\kappa_{1}\right)\left(R_{1}^{2}+R_{1}^{-2}\right)+2 \bar{X}\left(\Gamma_{1}-1\right)\right] \\
+\left(\Gamma_{3}+\kappa_{3}\right)\left\{2 X\left[R_{1}^{4}\left[\Gamma_{1}\left(\kappa_{2}-1\right)-\left(\kappa_{1}-1\right)\right]-\Gamma_{1}-\kappa_{1}\right]+\bar{X}\left[R_{1}^{2}\left[4-\kappa_{1}-\Gamma_{1}\left(4-\kappa_{2}\right)\right]+R_{1}^{6}\left(\Gamma_{1} \kappa_{2}-\kappa_{1}\right)\right]\right\}
\end{array}\right)}{\Gamma_{1}\left(\kappa_{2}+1\right)\left(\kappa_{3}+1\right)\left(R_{1}^{2}+R_{1}^{-2}\right) \xi^{2}},
\end{aligned}
$$$$
\left(|\xi| \geq R_{2}\right)
$$

where the two complex constants $\alpha$ and $\beta$ are defined as

$$
\begin{aligned}
\alpha & \left.\left.=\left(\Gamma_{3} \kappa_{2}+1\right) \mid X\left(\Gamma_{1}+\kappa_{1}\right)\left(R_{1}^{2}+R_{1}^{-2}\right)+2 \bar{X}\left(\Gamma_{1}-1\right)\right]+2 R_{2}^{-2}\left(1-\Gamma_{3}\right) \mid \bar{X}\left(\Gamma_{1}+\kappa_{1}\right)\left(R_{1}^{2}+R_{1}^{-2}\right)+2 X\left(\Gamma_{1}-1\right)\right] \\
& +R_{2}^{-4}\left(1-\Gamma_{3}\right)\left\{2 \bar{X}\left[R_{1}^{4}\left[\Gamma_{1}\left(\kappa_{2}-1\right)-\left(\kappa_{1}-1\right)\right]-\Gamma_{1}-\kappa_{1}\right]+X\left[R_{1}^{2}\left[4-\kappa_{1}-\Gamma_{1}\left(4-\kappa_{2}\right)\right]+R_{1}^{6}\left(\Gamma_{1} \kappa_{2}-\kappa_{1}\right)\right]\right\}, \\
\beta & =R_{2}^{4}\left(\Gamma_{3}-1\right)\left\{2 \bar{X}\left[R_{1}^{-4}\left[\Gamma_{1}\left(\kappa_{2}-1\right)-\left(\kappa_{1}-1\right)\right]-\Gamma_{1}-\kappa_{1}\right]+X\left[R_{1}^{-2}\left[4-\kappa_{1}-\Gamma_{1}\left(4-\kappa_{2}\right)\right]+R_{1}^{-6}\left(\Gamma_{1} \kappa_{2}-\kappa_{1}\right)\right]\right\} \\
& +2 R_{2}^{2}\left(\Gamma_{3}-1\right)\left[\bar{X}\left(\Gamma_{1}+\kappa_{1}\right)\left(R_{1}^{2}+R_{1}^{-2}\right)+2 X\left(\Gamma_{1}-1\right)\right]-\left(\Gamma_{3} \kappa_{2}+1\right)\left[X\left(\Gamma_{1}+\kappa_{1}\right)\left(R_{1}^{2}+R_{1}^{-2}\right)+2 \bar{X}\left(\Gamma_{1}-1\right)\right]
\end{aligned}
$$

It is found that $\phi_{3}(\xi)$ and $\psi_{3}(\xi)$ given by Eqs. (24) and (25) are analytic outside the circle $R_{2}$ (in 
view of the fact that $\xi^{2}-1 \neq 0$ for $|\xi| \geq R_{2}$ ) and meet the asymptotic condition in Eq. (9).

It can be easily proved that under a linearly distributed stress field at infinity, the internal stress field within an elliptical inclusion perfectly bonded to the surrounding matrix is always a linear function of the coordinates. The above obtained results demonstrate the interesting fact that the internal stress field within a three-phase elliptical inclusion can still keep a simple linear form if the remotely applied non-uniformly loadings satisfy the two conditions in Eq. (18).

\section{Discussions}

In this section several typical examples will be presented to illustrate the application of Eq. (18) to the design of three-phase elliptical inclusions when the matrix is subjected to a linearly distributed stress field at infinity.

3.1. The materials comprising the interphase layer and the matrix are identical $\left(\mu_{2}=\mu_{3}\right.$ and

$$
\left.\kappa_{2}=\kappa_{3}\right)
$$

In this case, Eqs. (18) becomes

$$
\begin{gathered}
\frac{B_{1}}{A_{1}}=\frac{R_{1}^{-6}\left(\Gamma_{1} \kappa_{2}-\kappa_{1}\right)+2 R_{1}^{-4}\left[\Gamma_{1}\left(\kappa_{2}-1\right)-\left(\kappa_{1}-1\right)\right]+R_{1}^{-2}\left[4-\kappa_{1}-\Gamma_{1}\left(4-\kappa_{2}\right)\right]-2\left(\Gamma_{1}+\kappa_{1}\right)}{\left(\Gamma_{1}+\kappa_{1}\right)\left(R_{1}^{2}+R_{1}^{-2}\right)+2\left(\Gamma_{1}-1\right)}, \\
\frac{B_{2}}{A_{2}}=\frac{R_{1}^{-6}\left(\kappa_{1}-\Gamma_{1} \kappa_{2}\right)+2 R_{1}^{-4}\left[\Gamma_{1}\left(\kappa_{2}-1\right)-\left(\kappa_{1}-1\right)\right]+R_{1}^{-2}\left[\Gamma_{1}\left(4-\kappa_{2}\right)-\left(4-\kappa_{1}\right)\right]-2\left(\Gamma_{1}+\kappa_{1}\right)}{\left(\Gamma_{1}+\kappa_{1}\right)\left(R_{1}^{2}+R_{1}^{-2}\right)-2\left(\Gamma_{1}-1\right)} .
\end{gathered}
$$

In particular, if the inclusion is a hole $\left(\Gamma_{1}<<1\right)$, Eqs. (27) and (28) become

$$
\begin{aligned}
& \frac{B_{1}}{A_{1}}=-\frac{1}{R_{1}^{4}}-\frac{2}{R_{1}^{2}}, \\
& \frac{B_{2}}{A_{2}}=\frac{1}{R_{1}^{4}}-\frac{2}{R_{1}^{2}} .
\end{aligned}
$$

On the other extreme end, if the inclusion is rigid ( $\left.\Gamma_{1}>>1\right)$, Eqs. (27) and (28) become

$$
\begin{aligned}
& \frac{B_{1}}{A_{1}}=\frac{\kappa_{2}}{R_{1}^{4}}-\frac{2}{R_{1}^{2}}, \\
& \frac{B_{2}}{A_{2}}=-\frac{\kappa_{2}}{R_{1}^{4}}-\frac{2}{R_{1}^{2}} .
\end{aligned}
$$

It is deduced from Eqs. (13) and (14) that

$$
\phi_{2}(z)=\phi_{3}(z)=\frac{X\left(\Gamma_{1}+\kappa_{1}\right)\left(R_{1}^{2}+R_{1}^{-2}\right)+2 \bar{X}\left(\Gamma_{1}-1\right)}{R^{2} \Gamma_{1}\left(\kappa_{2}+1\right)\left(R_{1}^{2}+R_{1}^{-2}\right)} z^{2},
$$


which implies that the mean stress in $S_{2}$ and $S_{3}$ is not disturbed. Thus the elliptical inclusion is harmonic when the remote non-uniform loadings satisfy the two restrictions given by Eqs. (27) and (28). It seems that the discussion on harmonic elliptical inclusions under non-uniform loadings has not been recorded in the literature. Previously harmonic elliptical inclusions were found only for remote uniform stresses [2, 7, 8]; whereas only non-elliptical harmonic inclusions were found for non-uniform loadings $[9,10]$.

Results similar to those derived in this subsection can also be obtained from Eq. (18) by letting $\rho=1$, or by assuming that the materials comprising the inclusion and the interphase layer are identical $\left(\mu_{1}=\mu_{2}\right.$ and $\left.\kappa_{1}=\kappa_{2}\right)$.

\subsection{Extremely compliant interface layer $\left(\Gamma_{1}>>1, \Gamma_{3}>>1\right.$ and $\left.\rho \neq 1\right)$}

In this case, Eq. (19) and (20) become

$$
\begin{aligned}
& f\left(R_{1}, \rho\right)=-\frac{2 \rho}{R_{1}^{2}}+\frac{\kappa_{2}(\rho+1)-2 R_{1}^{2}}{\rho R_{1}^{2}\left(\kappa_{2} R_{1}^{2}-2\right)+\kappa_{2} R_{1}^{4}}, \\
& g\left(R_{1}, \rho\right)=-\frac{2 \rho}{R_{1}^{2}}-\frac{\kappa_{2}(\rho+1)+2 R_{1}^{2}}{\rho R_{1}^{2}\left(\kappa_{2} R_{1}^{2}+2\right)+\kappa_{2} R_{1}^{4}} .
\end{aligned}
$$

The above two equations together with Eq. (18) give us very simple formulas for determining the restrictions on the remote non-uniform loadings when the interphase layer is much more compliant than both the inclusion and the matrix.

\subsection{Relatively rigid interphase layer $\left(\Gamma_{1}<<1, \Gamma_{3}<<1\right.$ and $\left.\rho \neq 1\right)$}

In this case, Eqs. (19) and (20) become

$$
\begin{aligned}
& f\left(R_{1}, \rho\right)=-\frac{2 \rho}{R_{1}^{2}}-\frac{\kappa_{3}\left(\rho+1+2 R_{1}^{2}\right)}{\rho R_{1}^{2}\left(R_{1}^{2}+2\right)+R_{1}^{4}}, \\
& g\left(R_{1}, \rho\right)=-\frac{2 \rho}{R_{1}^{2}}+\frac{\kappa_{3}\left(\rho+1-2 R_{1}^{2}\right)}{\rho R_{1}^{2}\left(R_{1}^{2}-2\right)+R_{1}^{4}} .
\end{aligned}
$$

The above two equations together with Eq. (18) also give us very simple formulas for determining the restrictions on the remote non-uniform loadings when the interphase layer is much stiffer than both the inclusion and the matrix.

\subsection{A three-phase circular inclusion $\left(R_{1} \rightarrow \infty\right)$}

In this case, Eqs. (19) and (20) reduce to

$$
R_{1}^{2} f\left(R_{1}, \rho\right)=R_{1}^{2} g\left(R_{1}, \rho\right)=-2 \rho-\frac{2(1-\rho)\left(\Gamma_{1}+\kappa_{1}\right)\left(\Gamma_{3}+\kappa_{3}\right)}{\rho^{2}\left(1-\Gamma_{3}\right)\left(\Gamma_{1} \kappa_{2}-\kappa_{1}\right)+\left(\Gamma_{1}+\kappa_{1}\right)\left(\Gamma_{3} \kappa_{2}+1\right)} .
$$

\section{Conclusions}

Similar to the case of uniform loading [2], confocal elliptical interfaces are used in the present design to achieve an internal stress field of linear form under a linearly distributed stress field at 
infinity. Two conditions given in Eq. (18) are derived that ensure that the internal field is a linear function of the two coordinates $x$ and $y$. These two conditions can be considered as restrictions on the remote non-uniform stress field for given material and geometric parameters of the composite. The specific expressions of the two conditions, which are generally rather tedious, will become very concise for the four special cases: (i) the interphase and the matrix have the same elastic constants; (ii) the interphase layer is extremely compliant; (iii) the interphase layer is rather stiff; (iv) the three-phase inclusion is circular.

\section{Acknowledgements}

This work is supported by the National Natural Science Foundation of China (Grant No: 11272121), the Innovation Program of Shanghai Municipal Education Commission, China (Grant No: 12ZZ058), and the National Basic Research Program of China (Grant No. 2009CB623204).

\section{References}

[1] C.Q. Ru, Effect of interphase layers on thermal stresses within an elliptical inclusion. J Appl Phys, 84 (1998) 4872-4879.

[2] C.Q. Ru, Three-phase elliptical inclusions with internal uniform hydrostatic stresses. J Mech Phys Solids, 47 (1999) 259-273.

[3] J.C. Luo, C.F. Gao, Stress field of a coated arbitrary shaped inclusion. Meccanica, 46 (2011)1055-1071.

[4] X. Wang, X.L. Gao, On the uniform stress state inside an inclusion of arbitrary shape in a three-phase composite. Z Angew Math Phys, 62 (2011)1101-1116.

[5] X. Wang, P. Schiavone, Three-phase inclusions of arbitrary shape with internal uniform hydrostatic stresses in finite elasticity. ASME J Appl Mech, 79 (2012) art. no. 041012 (6 pages).

[6] N.I. Muskhelishvili, Some Basic Problems of the Mathematical Theory of Elasticity, Groningen, Noordhoff, 1953.

[7] G.S. Bjorkman, R. Richards, Harmonic holes-an inverse problem in elasticity. ASME J Appl Mech, 43 (1976) 414-418.

[8] L.T. Wheeler, The problem of minimizing stress concentrations at a rigid inclusion. ASME J Appl Mech, 52 (1985) 83-86.

[9] G.S. Bjorkman, R. Richards, Harmonic holes for nonconstant fields. ASME J Appl Mech, 46 (1979) 573-576.

[10]G.F. Wang, P. Schiavone, C.Q. Ru, Harmonic shapes in finite elasticity under nonuniform loading. ASME J Appl Mech, 72 (2005) 691-694. 